\title{
A GLOBAL SHAPE MODEL FOR SATURN'S MOON ENCELADUS FROM A DENSE PHOTOGRAMMETRIC CONTROL NETWORK
}

\author{
M. T. Bland*, L. A. Weller., D. P. Mayer, B. A. Archinal \\ Astrogeology Science Center, U.S. Geological Survey, 2255 N. Gemini Dr., Flagstaff, AZ 86001 (mbland@usgs.gov)
}

Commission III, ICWG III/II

KEY WORDS: Enceladus, Shape Model, Topography, Photogrammetry

\begin{abstract}
:
A planetary body's global shape provides both insight into its geologic evolution, and a key element of any Planetary Spatial Data Infrastructure (PSDI). NASA's Cassini mission to Saturn acquired more than 600 moderate- to high-resolution images $(<500 \mathrm{~m} /$ pixel) of the small, geologically active moon Enceladus. The moon's internal global ocean and intriguing geology mark it as a candidate for future exploration and motivates the development of a PSDI. Recently, two PSDI foundational data sets were created: geodetic control and orthoimages. To provide the third foundational data set, we generate a new shape model for Enceladus from Cassini images and a dense photogrammetric control network (nearly 1 million tie points) using the U.S. Geological Survey's Integrated Software for Imagers and Spectrometers (ISIS) and the Ames Stereo Pipeline (ASP). The new shape model is near-global in extent and gridded to $2.2 \mathrm{~km} /$ pixel, $\sim 50$ times better resolution than previous global models. Our calculated triaxial shape, rotation rate, and pole orientation for Enceladus is consistent with current International Astronomical Union (IAU) values to within the error; however, we determined a new prime meridian offset $\left(W_{o}\right)$ of $7.063^{\circ}$. We calculate Enceladus' long-wavelength topography by subtracting the best-fit triaxial ellipsoid from our shape model. The result is comparable to previous global models but can resolve topographic features as small as 5-7 km across in certain areas. To evaluate the spatially varying quality of the model, we calculate the point density (variable from 5 to more than 50 points per pixel), normalized median absolute deviation of the points within each pixel (typically less than $100 \mathrm{~m}$ ), and the minimum expected vertical precision of each point (ranging from $29 \mathrm{~m}$ to $2 \mathrm{~km}$ ).
\end{abstract}

\section{INTRODUCTION}

\subsection{The importance of planetary shape and topography}

The global shape and topography of a planetary body provides insight into its interior structure, orbital dynamics, and thermal history. Characterizing a body's shape is therefore necessary for understanding its evolution over time. On a more practical level, topography is also critical to the development of numerous higher order data products (e.g., orthoimages), and along with geodetic control and orthoimages, constitutes one of the three foundational data sets necessary for a Planetary Spatial Data Infrastructure (PSDI) (Laura et al. 2017). PSDI is the collection of spatial data, access mechanisms, standards, policies, and data users that enables effective application of spatial data to scientific problems (Laura et al. 2018). The three foundational products can be used in combination with other data sets to generate numerous higher order spatial framework data. An effective PSDI must also include a rigorous characterization of product quality and uncertainty for all data products.

Here we describe a new global shape and topography (i.e., the deviation in shape from the best-fit ellipsoid) model for Saturn's moon Enceladus, which provides the final element of a PSDI for this moon (see section 1.2). In section 2, we describe our photogrammetric approach to shape model generation, which conceptually follows that of Archinal et al. (2005) and Becker et al. (2016), who applied similar techniques to the Moon and Mercury, respectively. Our results are described in section 3, and a characterization of the spatially variable quality of the shape model follows in section 4 . The shape model is publicly available as both a point cloud and gridded (interpolated) products at the Annex of NASA's Planetary Data System (PDS) Cartography and Imaging Science Node, which is supported by the U.S. Geological Survey (USGS), where we also provide updated pointing kernels (ck), and additional metadata (measures of quality).

\subsection{Why Enceladus?}

Despite its small size (251 km in radius), Saturn's moon Enceladus is geologically active, with plumes of ice and gas emanating from four warm, parallel fractures at its south pole (Hansen et al. 2006, Porco et al. 2006, Spencer et al. 2006) (Fig. 1). Salts detected in the plume by NASA's Cassini spacecraft suggest the fractures are conduits to a liquid reservoir (Postberg et al. 2009) at a depth of just 4-14 km (Hemingway and Mittal 2019). Detection of a large physical libration strongly suggests that the liquid reservoir is global in extent (Thomas et al. 2016) with an average ice shell thickness of 20-30 km (McKinnon 2015; Hemingway and Mittal 2019). Although the south pole is the locus of current activity, much of Enceladus' surface is geologically young, as indicated by extensive tectonic deformation and low impact crater density (Crow-Willard and Pappalardo 2015). Many of its craters have been extensively modified, indicating unusually high heat flow in Enceladus' past (Bland et al. 2012). The current elevated heat flow at the south pole, global ocean, and past periods of high heat flow must be maintained by tidal heating, but the exact mechanism by which sufficient heat is generated within Enceladus remains unclear (e.g., Meyer and Wisdom 2007, Roberts and Nimmo 2008, Roberts 2015, Souček et al. 2019).

Given Enceladus' remarkable geology, its status as a confirmed ocean world, and its tendency to spew its interior into space (where it can "easily" be sampled by spacecraft), the moon is a

\footnotetext{
* Corresponding Author
} 
focus of scientific interest and a target for future exploration. NASA's Cassini mission returned a wealth of image data of Enceladus (section 2.2). Unfortunately, inaccurate knowledge of the spacecraft position and pointing when the images were acquired make the data challenging to use: image locations on the surface can be inaccurate by several to tens of kilometers. This issue can be addressed by the development of a Planetary Spatial Data Infrastructure (PSDI) to enable the scientific community to effectively utilize geospatially accurate data returned by Cassini.

Previously, Bland et al. (2018) described a photogrammetric control network for Enceladus that included a total of 586 Cassini Imaging Science Subsystem (ISS) (Porco et al. 2004) images of Enceladus (additional images were added later to bring the total number of images to 621 ). That work provided two of the three foundational PSDI products: photogrammetric control and orthorectified images (section 1.1). We distinguish between photogrammetric control, in which there is no "ground," and geodetic control, which uses laser altimetry data or some other means to determine absolute control. To date, no such data have been acquired for Enceladus, so photogrammetric control is the highest level of control achievable. We note that the control network of Bland et al. (2018), following IAU recommendations (Archinal et al. 2018, Table 2), did fix the location of the crater Salih at $5^{\circ} \mathrm{W}$, which defines Enceladus' longitude system and provides one geodetic reference point. We also note that the orthoimages produced by Bland et al. (2018) are projected to Enceladus' triaxial shape rather than local topography.

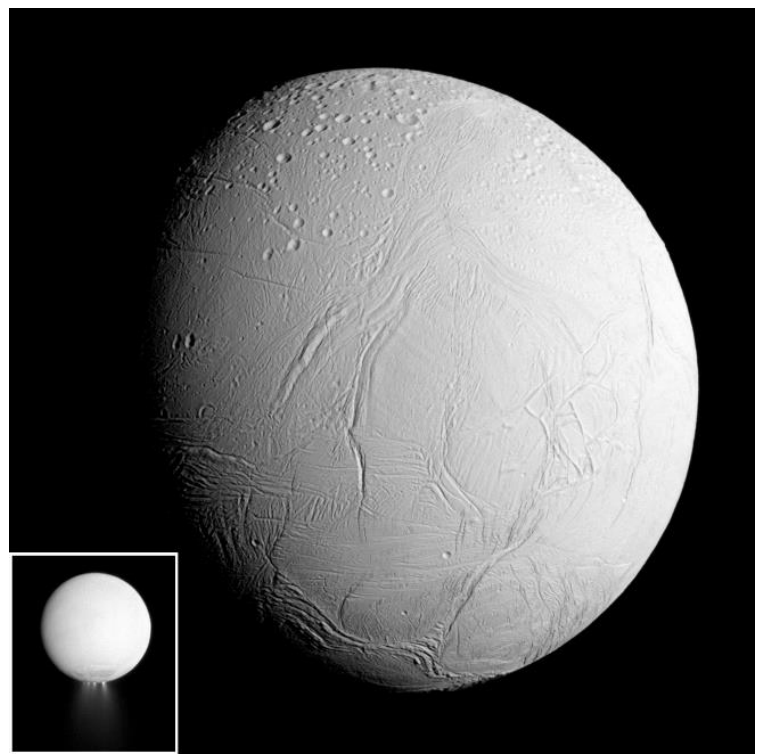

Figure 1: The surface of Saturn's small moon Enceladus $(251 \mathrm{~km}$ radius) is highly deformed, indicating a complex geologic history. Plumes of dust (mostly ice) and gas (mostly water vapor) emanate from its south pole (inset). Images were acquired by the ISS on NASA's Cassini spacecraft and are publicly available through the Planetary Data System (PDS).

\subsection{Previous shape models}

Several global, or semi-global topographic data sets have previously been created for Enceladus. These data sets were derived from two different techniques: limb fitting, and stereo imaging. Porco et al. (2006) provided the first measurements of Enceladus triaxial shape from Cassini images of Enceladus' limb (the approach was described more fully by Thomas et al. (2007)), and these measurements were subsequently updated by Thomas (2010) at the end of Cassini's nominal mission, with the only change being a decrease in the uncertainty in the tidal $(a)$ semi- axis. The Thomas (2010) analysis derived the triaxial shape of Enceladus ( $a, b, c$ semi-axes) to within 200-300 m, but did not report on Enceladus' regional or local topography.

Schenk and McKinnon (2009) provided the first semi-global topography model for Enceladus by using stereo imaging to derive topography for $\sim 50 \%$ of Enceladus' surface. The model has a relatively small horizontal pixel scale of $200-950 \mathrm{~m} /$ pixel and a stated vertical precision of 50 to $140 \mathrm{~m}$. Significantly, their analysis identified six large-scale depressions, 800-1500 m deep, that are not correlated with obvious surface geology, and confirmed the presence of a previously identified south polar depression (cf. Porco et al. 2006; Collins and Goodman 2007). The depressions might correspond to thin regions of the ice shell (Schenk and McKinnon 2009) or local regions of convectiondriven compaction (Besserer et al. 2013).

The first truly global shape model of Enceladus was provided by Nimmo et al. (2011), who performed a spherical harmonic expansion of the Thomas (2010) limb data up to degree 8 . Although the degree- 8 expansion corresponds to a spatial scale of just $45^{\circ}$ or $\sim 200 \mathrm{~km}$ at the equator $(\lambda \sim 2 \pi R / L$, where $R$ is Enceladus' mean radius of $251 \mathrm{~km}$, and $L$ is the harmonic degree), the model enabled a more detailed evaluation of Enceladus' long-wavelength global topography. Where they overlap, the shape derived by Nimmo et al. (2011) is in general agreement with the stereo model of Schenk and McKinnon (2009) and generally confirms, with some variation, the existence of the large-scale topographic depressions.

Using a similar approach, but with the benefit of a more extensive data set, and the additional use of control points, Tajeddine et al. (2017) performed a spherical harmonic expansion of Enceladus limb data to degree 16, improving the spatial "resolution" by a factor of 2 relative to the Nimmo et al. (2011) model (to $22.5^{\circ}$ or $\sim 100 \mathrm{~km}$ at the equator). The results are largely consistent with the lower degree shape model of Nimmo et al. (2011) and again reveal a chain of topographic basins that Tajeddine et al. (2017) argue are evidence of a relic equator, and thus true polar wander. However, the two models are different enough in their details that analysis of their implications for Enceladus' interior structure yields modestly different results (Hemingway and Mittal 2019).

The existing shape models for Enceladus thus have their strengths and weaknesses. The spherical harmonic expansions of Nimmo et al. (2011) and Tajeddine et al. (2017) are global in extent, but relatively low resolution. In contrast, the stereo model of Schenk and McKinnon (2009) is 2 orders of magnitude higher resolution (at least locally) but covers just a portion of Enceladus. Here we describe a new shape model for Enceladus created with a photogrammetric approach that balances resolution and coverage, providing a shape model that is nearly global $(>92 \%$ coverage), relatively high resolution (2 pixel/degree or 2.2 $\mathrm{km} /$ pixel at the equator), and vertically precise (root mean square radius uncertainty of $57 \mathrm{~m}$ ).

\section{METHODOLOGY}

\subsection{Overview}

We have created a global shape model of Enceladus using 625 Cassini ISS images of Enceladus following the conceptual approach applied to Mercury MESSENGER data by Becker et al. (2016). We first established a dense network of image tie points and photogrammetrically solved for point latitude, longitude, and radius using a least squares bundle adjustment. The resulting point cloud was then interpolated to a regularly gridded $2.5 \mathrm{D}$ 
global shape model. We subtracted a best-fit triaxial ellipsoid from the model to reveal the moon's long-wavelength topography (local deviation from the ellipsoid). The data set and each of these steps are described in more detail below. We describe the shape model quality in section 4 .

\subsection{The Cassini image data set}

NASA's Cassini mission spent 13 years (2004 to 2017) in the Saturn system. The long mission duration and high-performing spacecraft enabled 23 targeted flybys of Enceladus. These flybys, and an additional $\sim 30$ more-distant flybys, returned more than 22,000 images, including more than 600 images with a pixel scale better than $500 \mathrm{~m} /$ pixel, from Cassini's ISS cameras. The ISS included a $2-\mathrm{m}$ focal length, $0.35^{\circ}$ field of view (FOV) narrow angle camera and a $0.2-\mathrm{m}$ focal length $3.5^{\circ} \mathrm{FOV}$ wide angle camera that shared a 1024 by 1024 pixel charged couple device (CCD) detector (Porco et al. 2004).

Although Cassini achieved global image coverage, the orbital dynamics of the mission, the scientific focus on Enceladus' south pole, and the seasonal illumination constraints resulted in highly variable image quality across the satellite. For example, only a few images of the northern polar region were acquired due to it being dark during northern winter until late in the extended mission. Image coverage is also sparse in the trailing hemisphere due to the orbital dynamics of the mission: Cassini only flew over these areas early in the mission. Even where multiple flybys returned repeat coverage of the same areas, differences in flyby altitude and the location of the closest approach of each flyby resulted in large differences in image pixel scale at the same location. Illumination and viewing geometry also varied substantially between flybys. Many images include the moon's limb, terminator, or both (incidence and emission angles up to $90^{\circ}$ ) and phase angles reach a maximum of $178^{\circ}$.

The multiple-flyby nature of the data set poses a substantial challenge for the image-to-image matching that is required to create a photogrammetric control network for the purposes of improving image locations and/or deriving shape information. High incidence angles create problematic shadows and high emission angles distort features and compromise measurement accuracy. Changes in illumination also substantially affect the appearance, and even visibility, of surface features (see Bland et al. 2018 for examples). Matching images with substantially different pixel scales is especially challenging.

Despite these challenges, Bland et al. (2018) created a relatively sparse (compared to the present work) tie-point network and photogrammetrically updated the location of 586 images with pixel scale between 50 and $500 \mathrm{~m} /$ pixel, phase angle less than $120^{\circ}$, and filter settings of CLR, GRN, IR3, and UV3 (Porco et al. 2004). Least squares bundle adjustment resulted in root-meansquare (RMS) residuals of 0.45 pixels, corresponding to ground point uncertainties of 66,51 , and $46 \mathrm{~m}$ in latitude, longitude, and radius, respectively. Subsequently, 35 images of the north pole acquired late in the Cassini mission were tied to the network, creating a global set of 621 images. Additional high-resolution images were then tied to the global basemap on an image-byimage basis. The resulting mosaics are available through the USGS Astropedia data portal at https://astrogeology.usgs.gov/search/map/Enceladus/Cassini/En celadus_Cassini_mosaic_global_110m. These updated images form the basis for the work described here. Although points from the sparse network itself were not utilized, the updated image locations facilitated more efficient matching during construction of our new dense tie-point network.

\subsection{The dense photogrammetric control network}

To create a dense, global network of tie points we used an imageby-image approach such that every image in the data set has its own individual tie-point network. These 621 individual networks were then merged to create the global network (Fig. 2). Four additional images were subsequently added to provide additional coverage in the trailing hemisphere, bringing the total number of images used to 625 .

For every image, we first established a regularly spaced grid of tie points with an initial density of one point every 20 line/sample (Fig. 2b,c). Increasing the density lead to computational difficulty due to the network size. Automated tie-point matching was performed between the reference image in question and every other overlapping image. To do so, we used an area-based approach with a maximum correlation algorithm via ISIS's pointreg application (Garcia et al. 2015). A weighted centroiding approach was used to improve the match to subpixel accuracy. Matches below a Goodness-of-Fit threshold were ignored, and false matches were removed based on bundle adjustment residuals (section 2.4). Every successfully matched image provided an image "measure" for that tie point. Most tie points are tied to multiple other images, providing numerous measures and substantial network "depth," which yields a photogrammetrically robust solution (Bland et al. 2018) and increases stereo strength. Because every image is associated with its own small network, matching between each overlapping image pair is attempted twice. That is, image-B is included in image-A's network and image-A is include in image-B's network. Tie points are identified in both directions but because images often only partially overlap and are of different resolution, points from each network occur at different line/sample locations and are therefore generally unique. The 625 individual image networks were combined into a single global network using ISIS's cnetmerge application. This approach has the benefit of correlating tie-point density with depth of image coverage at a location. That is, where many images overlap, point density is high, and where image coverage is limited, point density is lower. Spatial variation in tie-point density is therefore naturally indicative of local data density (although not necessarily quality, see section 4). The resulting tie-point network contains 892,457 points and more than 30 million image measures. The network is nearly $\sim 90 \mathrm{x}$ more spatially dense than the network used by Bland et al. (2018).

Our approach to control network generation differs from that of Becker et al. (2016) who used the OpenCV library (opencv.org) of feature-based matching algorithms (as implemented in ISIS's findfeatures application) to create a dense network for Mercury. We found that our initial attempts at feature-based matching did not provide adequate spatial coverage (i.e., some areas had insufficient tie-point density), and that matching images with highly variable illumination conditions were more challenging then when area-based matching was used. However, given the large number of detector and extractor algorithms available, utilizing feature-based matching on challenging flyby data sets warrants additional investigation.

\subsection{Bundle adjustment}

Once the global network of tie points was established and point matching was complete, we performed a least squares bundle adjustment (Brown 1958) using the ISIS application jigsaw (Edmundson et al. 2012) to update camera pointing and image locations. All observations were weighted equally. In the 
adjustment, we solved for the camera pointing (three rotation angles) and the 3D coordinates (latitude, longitude, and radius) of each tie point in the network. We did not solve for spacecraft position, as doing so added additional parameters without improving the solution. A priori camera pointing (angles) was constrained to $\pm 0.5^{\circ}$, and a priori point radii were constrained to $1000 \mathrm{~m}$. In our final solution, we also constrained point latitude and longitude to $1000 \mathrm{~m}$. This additional constraint improved convergence of our bundle solution and decreased point uncertainties. We used jigsaw iteratively to identify and remove false matches from the network. Measures with very high residuals after bundle adjustment often indicate a false match. These measures are removed from the network, and a new bundle adjustment is performed. This process is repeated until either all point residuals are within an acceptable tolerance or visual inspection indicates quality matches despite some high individual residuals. Our final bundle adjustment resulted in RMS ground point uncertainties of $37 \mathrm{~m}, 36 \mathrm{~m}$, and $57 \mathrm{~m}$, in latitude, longitude, and radius, respectively. The total RMS uncertainty in image location was 0.3 pixels. A more detailed description of the shape model quality is provided in section 4 .

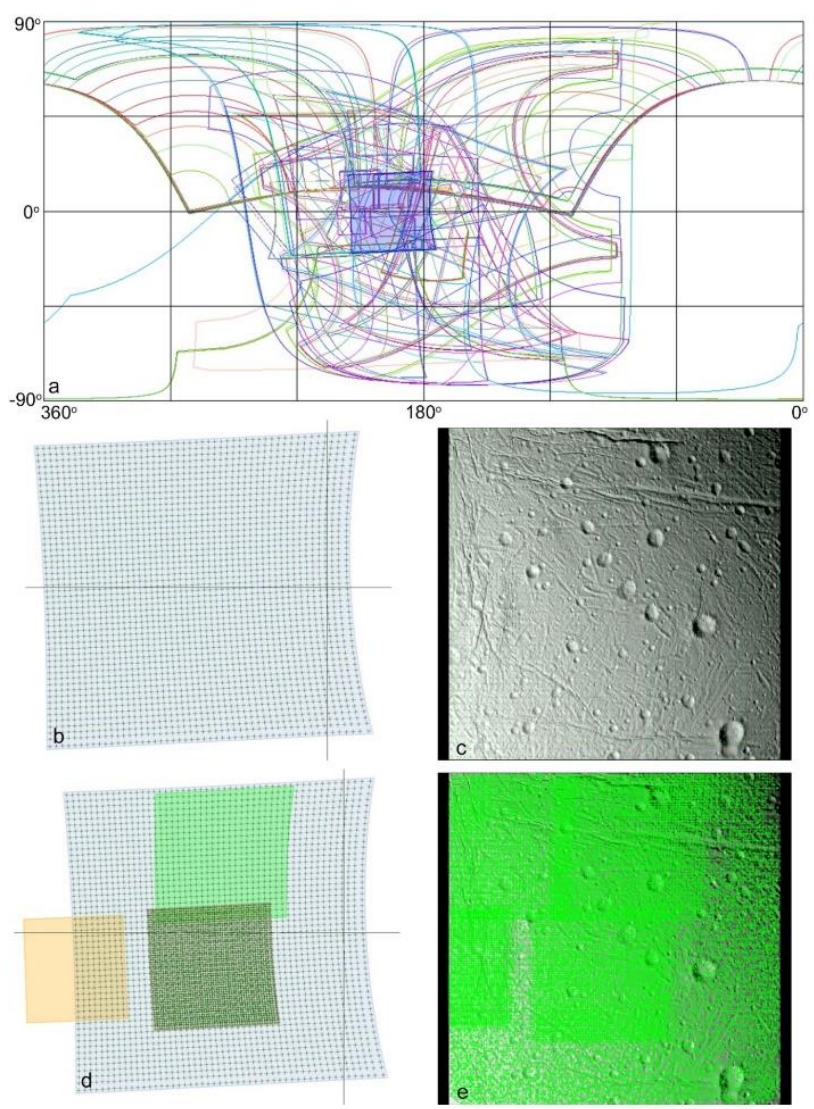

Figure 2: Illustration of our approach to control network generation for Enceladus. a) The blue rectangle is the footprint of a single ISS image (our example image is $167 \mathrm{~km}$ across). Also shown are the outlines of every image footprint that overlaps our example image (equirectangular projection, west longitude). b) Each image has its own line/sample tie-point network (green points on image footprint, which is the highlighted footprint in 'a'). c) The same tie points as seen on the actual unprojected image. d) Merging two networks locally increases network density. e) The final distribution of tie points on our example image from all the individual merged networks (see panel a).

Once a final bundle adjustment has been performed, the collection of 3D coordinates of each tie point (latitude, longitude, radius) constitute a point cloud that defines Enceladus' shape. As a final step, we calculate the minimum expected vertical precision (EP) of each point (see section 4) and filter out any points with EP greater than $\sim 2 \mathrm{~km}$, which is the maximum topographic relief on Enceladus. Snapshots of the point cloud are shown in Fig. 3.
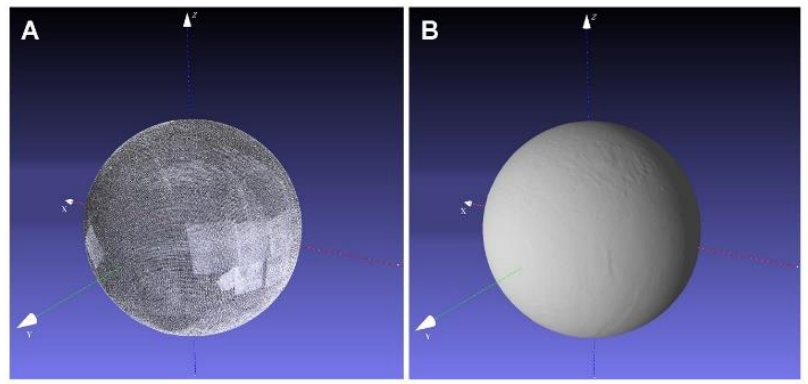

Figure 3: Visualization of the 3D point cloud. A) The individual points. Regions with more points appear brighter. B) Mesh rendering of the point cloud performed in Meshlab (Cignoni et al. 2008).

\subsection{The triaxial shape solution}

The ISIS jigsaw application can also be used to calculate the bestfit triaxial shape and orientation of Enceladus. Doing so requires specifying an initial "guess" and uncertainty values for each value. In total, we solved for the triaxial shape ( $a, b, c$ semi-axes), average radius, spin rate, pole orientation (right ascension and declination), and prime meridian offset $\left(W_{o}\right)$. Because images were acquired over a range of true anomaly, we assumed the effect of libration on our solution is small. The results are described in section 3 .

\subsection{Interpolation to a 2.5D global shape model}

The point cloud resulting from our photogrammetric solution is not uniform in spatial density. We therefore interpolated to a uniformly gridded $2.5 \mathrm{D}$ shape model using the Ames Stereo Pipeline (ASP) point2dem application (Beyer et al. 2018). We chose a grid spacing of 2 pixel/degree (ppd) $(2.2 \mathrm{~km} / \mathrm{pixel}$ at the equator) based on an analysis of areal coverage and point density (points per pixel) (Figure 4). At 2 ppd we achieve 92.5\% coverage of Enceladus. Decreasing the resolution to $1 \mathrm{ppd}$ reduces the resolution by a factor of $2(4.4 \mathrm{~km} / \mathrm{pixel})$ but provides only a small increase in coverage (from $92.5 \%$ coverage to $96.3 \%$ ). Alternatively, increasing the resolution to $3 \mathrm{ppd}$ decreases the coverage by 7 percentage points (from $92.5 \%$ coverage to $85.6 \%$ ), which we believe is unacceptably low for a global product. We also find that a grid spacing of 2 ppd yields an average point density of 10.6 points per pixel (Fig. 4b), which enables more robust evaluation of point statistics in the gridded product (section 4). Using a resolution of 3 ppd decreases the number of points per pixel by a factor of 2 . A mean density of 1 point per pixel is achieved at $6 \mathrm{ppd}(733 \mathrm{~m} / \mathrm{pixel})$ but such a high resolution only provides coverage over $57 \%$ of Enceladus. Thus, a grid spacing of 2 ppd provides a good balance of resolution, coverage, and point density.

Radius values at each pixel were determined by a Gaussian weighted average of the points within each pixel. We used a 1pixel search window in point $2 \mathrm{dem}$, which eliminates any smoothing that results from including points outside the pixel in the average. No additional gap-filling or smoothing was applied. The gridded shape model is shown in Fig. 5. 

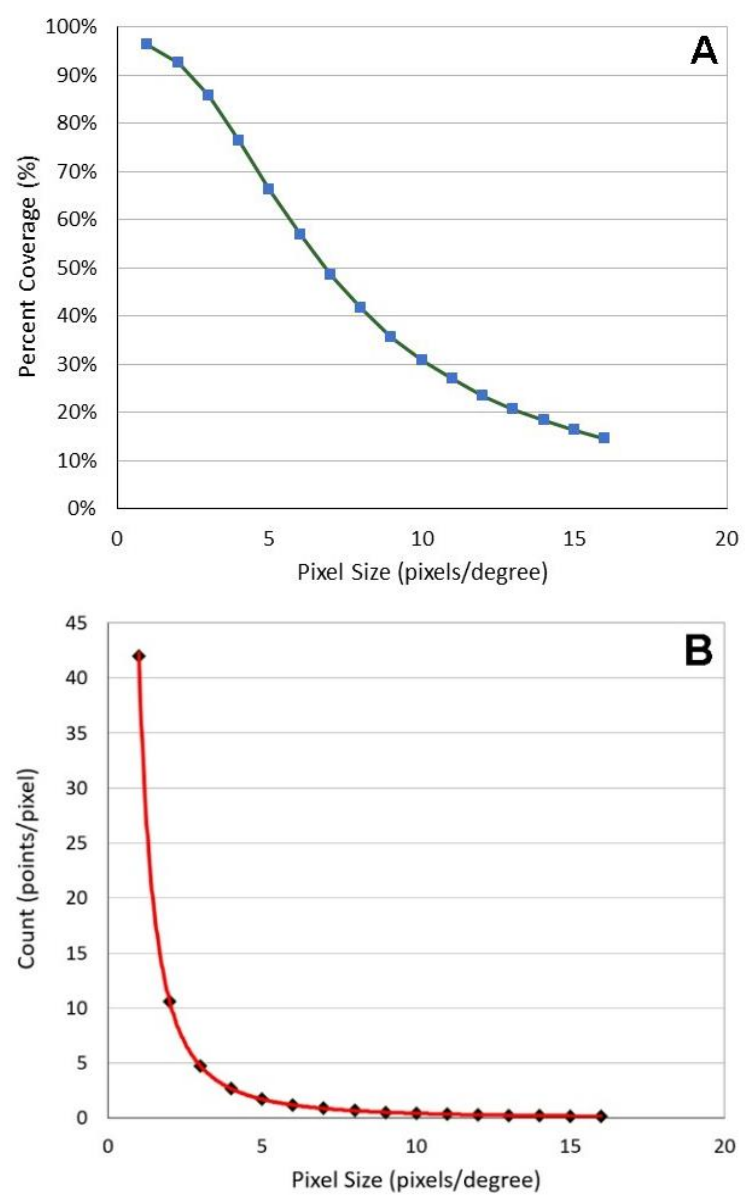

Figure 4: Statistics used for determining shape model interpolation. A) The percent coverage of Enceladus as a function of pixel size (larger pixels result in more complete coverage). B) The number of points per pixel as a function of pixel size.

\subsection{Calculating Enceladus' long-wavelength topography}

In addition to Enceladus' global shape, which is strongly triaxial, we also calculated the long-wavelength topography, which we define as local deviation in radius from the best-fit triaxial shape. To do this, we calculate the expected radius at each point (latitude/longitude) in our point cloud assuming the smooth triaxial shape derived in section 2.5 and then subtract it from the local ("true") radius value for that point derived from our bundle solution. The result is a set of latitude/longitude/elevation points, where the elevation is relative to the best-fit ellipsoid. As with the shape, we interpolated the topography data to a fixed grid using point 2 dem with a 2 ppd grid size and Gaussian weighting. The gridded topography is shown in Fig. 6.

\section{BASIC CHARACTERISTICS OF ENCELADUS' SHAPE AND TOPOGRAPHY}

\subsection{Enceladus' triaxial shape}

Direct interpolation of the point cloud to a gridded 2.5D product yields a nearly continuous shape model for Enceladus (Fig. 5). Data gaps (mostly in the trailing hemisphere) appear as black spots or regions where the underlying grayscale basemap shows through. As expected, the model is dominated by Enceladus' triaxial shape which stems from the tidal distortion of the moon by Saturn and results in a long tidal axis $(a)$ that is oriented toward/way from Saturn, a short polar axis $(c)$, and an intermediate axis $(b)$. The jigsaw solution (section 2.7) yields $a$, $b$, and $c$ semi-axes of $256.3 \mathrm{~km}, 251.2 \mathrm{~km}$, and $248.3 \mathrm{~km}$, respectively. The values are consistent with the previous determination by Bland et al. (2018), who used a similar method but much sparser network, and are within the uncertainty of the current IAU values (Archinal et al. 2018, their table 5).
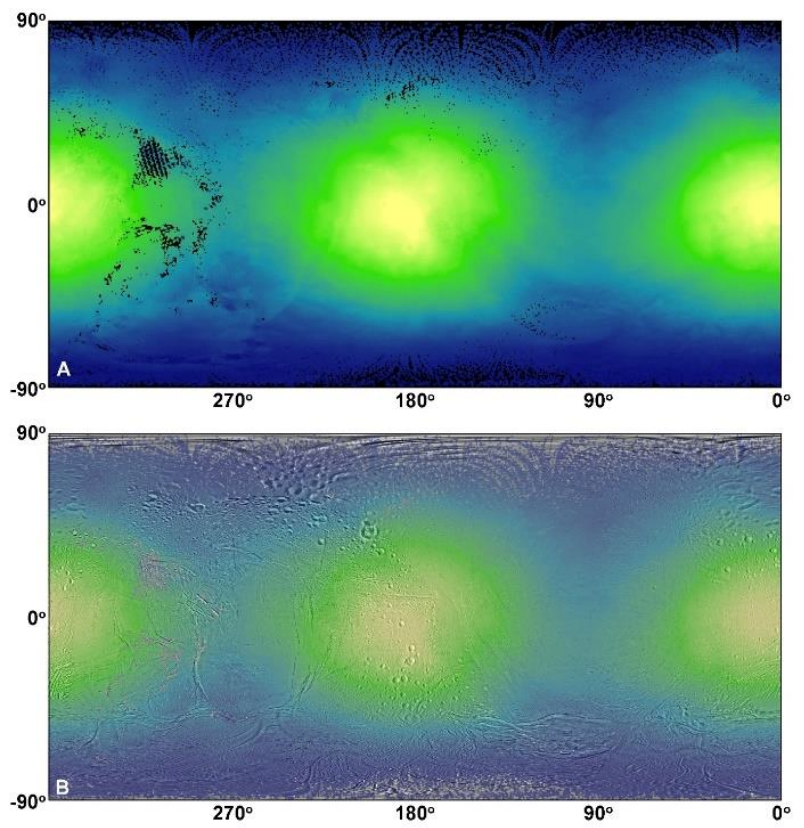

Figure 5: A) The gridded 2.5D global shape model of Enceladus. The model is dominated by the moon's triaxial shape. Values are relative to a mean sphere with radius $251.5 \mathrm{~km}$ (total range of +5300 to $-3900 \mathrm{~m}$ ): yellows are high, and blues are low. Equirectangular projection in west longitude. B) As in A but overlain on an image mosaic of Enceladus.

Again using jigsaw, we calculate a spin rate that is effectively identical to the IAU value (262.73198 vs. 262.7318996 degrees/day), and we find a small modification to the pole position (J2000.0 right ascension and declination of $40.56^{\circ}$ and $83.56^{\circ}$ compared to the IAU values of $40.66^{\circ}$ and $83.52^{\circ}$ ). Our new values are more similar to the IAU values than those reported in Bland et al. (2018). Jigsaw also yields $W_{o}$ of $6.088^{\circ}$, which is somewhat smaller than the IAU value of $6.32^{\circ}$ (Archinal et al. 2018, their Table 2). Following Bland et al. (2018), we also calculate $W_{o}$ using a more physical approach, in which we set a tie point within the center of the crater Salih (which is located at $5^{\circ} \mathrm{W}$ longitude and defines the Enceladus coordinate system) and determine the longitude adjustment necessary to maintain Salih's correct position. This approach yielded a $W_{o}$ of $7.063^{\circ}$, similar to that of the sparse network $\left(7.089^{\circ}\right)$ reported in Bland et al. (2018), but significantly larger than the IAU value. The reason for the inconsistency between the two approaches remains unclear and will require further validation of the jigsaw body solver.

\subsection{Long-wavelength topography}

Figure 6 shows our gridded 2.5D topographic model. Here the topography is relative to our best-fit ellipsoid. Several features are notable in the topography. The expected asymmetry (based on the earlier shape determination, e.g., Porco et al. (2006)) between the northern and southern hemispheres is apparent. The south pole is $200-400 \mathrm{~m}$ below the triaxial shape and $~ 500-1000$ $\mathrm{m}$ lower than the north pole. The model also clearly shows a chain of depressions stretching across the anti-Saturnian hemisphere from southeast to northwest. The deepest depression (near $150^{\circ}$ $\mathrm{W},-12^{\circ} \mathrm{S}$ ) sits $\sim 800 \mathrm{~m}$ beneath the reference ellipsoid, and more 
than $1 \mathrm{~km}$ below the topography directly to the west. The depressions become shallower moving to the northwest, with the northern-most depression (near $210^{\circ} \mathrm{W}, 45^{\circ} \mathrm{N}$ ) having a maximum depth of $\sim 500 \mathrm{~m}$. In our model, obvious basins do not extend farther north or west, although the region is modestly lower $(\sim 100 \mathrm{~m})$ than its surrounding. This contrasts with the lower-resolution model of Tajeddine et al. (2017), which identified a small, shallow basin extending farther west.

Our model also resolves a deep and extensive basin in the northern portion of the trailing hemisphere $\left(300^{\circ} \mathrm{W}, 28^{\circ} \mathrm{N}\right)$ that extends west to the sub-Saturnian hemisphere $\left(0^{\circ} \mathrm{W}\right.$ and $\left.50^{\circ} \mathrm{N}\right)$. The quality of the shape model is relatively poor in this region, with sparse data and limited stereo (section 4); however, the broad outline of the basin is easily distinguished. The basin in our model is $\sim 700 \mathrm{~m}$ deep. The region was identified as two separate basins by Schenk and McKinnon (2009), but this is likely due to a data gap in their model (where our data are also poor) Interestingly, the model of Tajeddine et al. (2017) also resolves a single deep basin centered at $330^{\circ} \mathrm{W}$ (farther west relative to our current model and that of Schenk and McKinnon (2009)) with much smaller longitudinal extent.
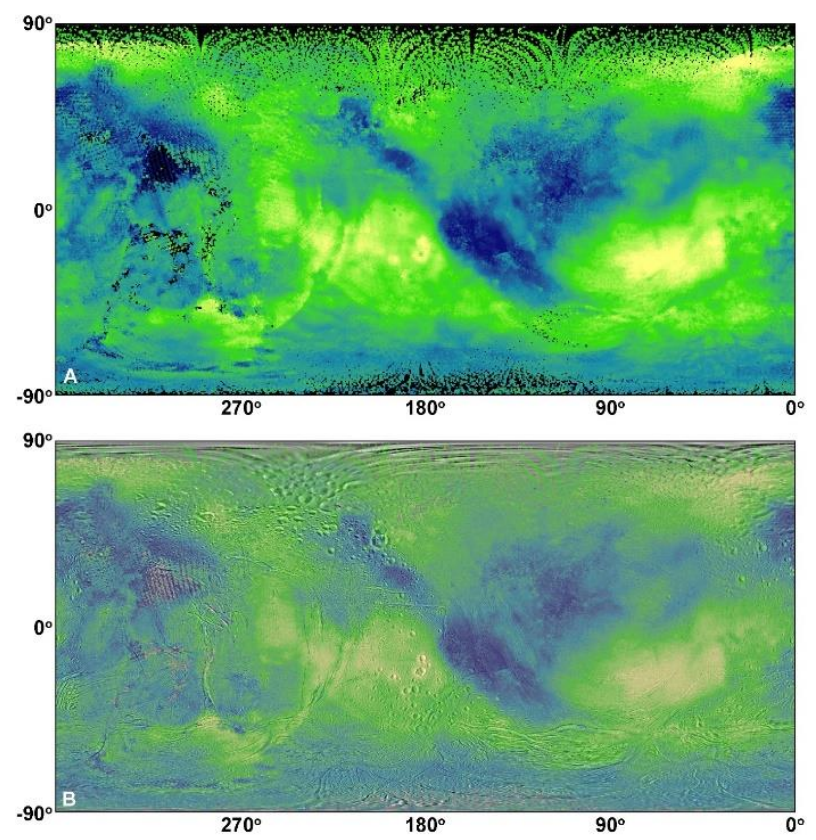

Figure 6: The gridded topography of Enceladus (values relative to the best-fit ellipsoid). Panels are as in Fig. 5.

The long-wavelength topography of Enceladus' leading hemisphere is also notable. The northwestern half consists of a broad depression $250-300 \mathrm{~km}$ in diameter and $\sim 600 \mathrm{~m}$ deep, whereas the southeastern half consists of a broad high that sits $\sim 800-1000 \mathrm{~m}$ above the reference. This large-scale topographic structure is not resolved in the Tajeddine et al. (2017) model, which shows a relatively flat region with a few small topographic depressions and highs. The region was not covered in the highresolution stereo model of Schenk and McKinnon (2009).

In some regions, our shape model clearly resolves smaller-scale topography associated with actual surface features. Relatively small craters $(\sim 7 \mathrm{~km}$ in diameter $)$ are easily distinguished near $180^{\circ} \mathrm{W}, 0^{\circ} \mathrm{N}$, even without the underlaying base image. We also resolve high-standing crater rims in these regions. Farther north, the rim of the large crater Dunyazad stands out above the depression to its west and south. Topography around the south polar terrain is also clearly associated with features, although the relationship is more complex. The band of ridges at $105^{\circ} \mathrm{W}$ is high standing, as is Cashmere Sulci: the pronounced set of ridges south of the deep trough Labtayt Sulci. Labtayt Sulci itself is also resolved, although the model is noisy in the region. Between those two sets of ridges, the shape model has a clear artefact, as indicated by an abrupt change in elevation across what appears to be an image seam. This type of artefact is rare in the model, and the root cause is not yet clear. Polar projections of the south pole reveal that the model resolves (barely) the large fractures associated with plume eruptions. The observations of such smallscale features reveal the power of this new data set.

\section{EVALUATING SHAPE MODEL QUALITY}

Generating foundational data sets is necessary for the construction of a PSDI; however, a measure of the quality of that data set is also necessary to help ensure that data are used appropriately. Below we describe measures of the quality of the shape model. Given the highly variable image data set from which it was derived (section 2.2), shape model quality is also highly spatially variable.

\subsection{Data density and variability}

One of the simplest measures of data quality is data density: literally how many points were included in each pixel. As discussed in section 2.2, image coverage of Enceladus is highly variable, resulting in highly variable point density. Figure $7 \mathrm{a}$ shows a map of the point density of the model, which ranges from 162 points per $2.2-\mathrm{km}$ pixel to 0 points per pixel. Point density is highest near the sub- and anti-Saturnian points, especially in the equatorial region near $210^{\circ} \mathrm{W}$ where typical point counts are 4060 . This region includes both a few relatively high-resolution images and numerous, overlapping lower-resolution images (Fig. 2). Point density outside of these regions (dark grey) are typically much lower: often just 5-10 points. The mean point density for the model is 10.6 points per pixel (mean is 7 points per pixel). Point density is a useful measure of where the data are but is of limited use in understanding the actual quality of the data. Pixels with high point density might still be low quality if, for example, all the images have similar viewing geometry (low stereo strength). Alternatively, pixels with low point density may be high-quality if all the points were derived from excellent stereo views.

To better assess the variability of the points within each pixel we calculated the normalized median absolute deviation (NMAD) (Fig. 7b), which is a robust measure of the statistical dispersion of the data (i.e., more resilient to outliers than, e.g., standard deviation). Yellow pixels indicate that the NMAD is less than the precision of our bundle solution $(57 \mathrm{~m})$. Blue pixels indicate NMAD > $100 \mathrm{~m}$, and thus highlight regions where point measurements are more variable. We note that some of this variability is "real": NMAD values are high near resolved craters (e.g., $200^{\circ} \mathrm{W}, 3^{\circ} \mathrm{N}$ ), which results from actual topographic variation. NMAD values are typically less than $250 \mathrm{~m}$, except for small isolated regions with limited data coverage.

\subsection{Evaluating stereo strength}

In general, stereo imaging was not a primary driver during the acquisition of Cassini ISS images. The availability of "good" stereo is therefore as much a function of happenstance as planning. In order to evaluate the spatial variability of the stereo strength of our model, we calculated the expected vertical precision (EP) of every pairwise combination of images that contribute to a given point in the model and report the minimum 
$\mathrm{EP}$ as a characteristic value for each point. We follow Kirk et al. (2003) and define EP $=\rho S /(p / h)$, where $S$ is the RMS pixel scale of the two images, $(p / h)$ is the parallax to height ratio of the images, and $\rho$ is the quality of the matching in fractions of a pixel. We conservatively take $\rho$ to be 1 (pixel, rather than subpixel matching) due to the often large disparity in pixel scale between the stereo images for each point. The result is that the EP reported here is likely an upper limit. The resulting minimum EP is shown in Fig. 8. The best $10 \%$ of the data have EP between 29 and 111 $\mathrm{m}$, and $70 \%$ of the data have EP $<200 \mathrm{~m}$. EP is highest at the poles where similar viewing geometry (south pole) and lack of data (north pole) severely limits stereo quality.
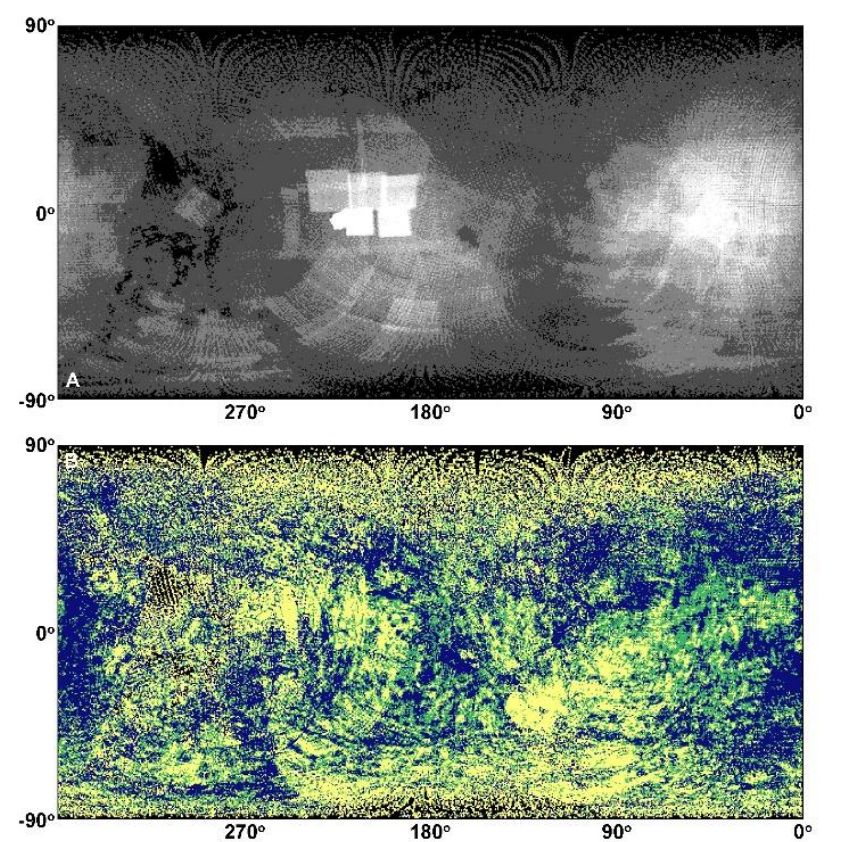

Figure 7: A) The number of points (from the point cloud) per pixel in the gridded product. White $>40$; grey shades in 4 bins from lightest to darkest, 31-40, 21-30, 11-20, 1-10; black is no data. B) The normalized median absolute deviation in three categories: 0-57 m (yellow), 57-100 m (green), and > $100 \mathrm{~m}$ (blue). Panels are as in Fig. 5.

\section{CONCLUSION}

We have generated a publicly available, global, high-resolution (relative to existing products) shape model for Saturn's moon Enceladus, which is the target of ongoing scientific research and future exploration. The spatially variable quality of the model has been well-characterized. The model provides the final foundational data product necessary for an Enceladus PSDI and demonstrates the applicability of the technique to future outer Solar System missions, such as NASA's Europa Clipper and the European Space Agency's Jupiter Icy moons Explorer (JUICE) mission.

\section{ACKNOWLEDGEMENTS}

L. Weller constructed the control network and performed the bundle solutions. D. Mayer generated the gridded products and calculated stereo strength. Tammy Becker generated the original sparse Enceladus network described here and in Bland et al. (2018). Any use of trade, firm, or product names is for descriptive purposes only and does not imply endorsement by the U.S. Government.

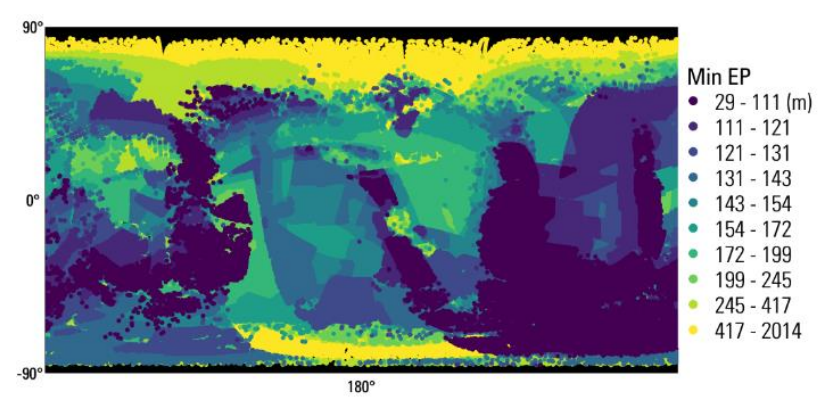

Figure 8: The minimum expected vertical precision at each point in the control network grouped in $10 \%$ bins. Panel as in Fig. 5.

\section{REFERENCES}

Archinal, B. A., Acton, C. H., A'Hearn, M. F., Conrad, A., Consolmagno, G. J., Duxbury, T., Hestroffer, D., Hilton, J. L., Kirk, R. L., Klioner, S. A., McCarthy, D., Meech, K., Oberst, J., Ping, J., Seidelmann, P. K., Tholen, D. J., Thomas, P. C., Williams, I. P., 2018: Report of the IAU working group on cartographic coordinates and rotational elements: 2015. Celest. Mech. Dyn Astr., 130:22, doi.org/10.1007/s10569-017-9805-3.

Archinal, B. A., M. R. Rosiek, R. L. Kirk, and B. L. Redding, 2006: The unified Lunar control network 2005. U.S. Geological Survey Open-File Report 2006-1367, 21 p. http://pubs.usgs.gov/of/2006/1367/.

Becker, K., J., Robinsons, M. S., Becker, T. L., Weller, L. A., Edmundson, K. L., Neumann, G. A., Perry, M. E., Solomon, S. C., 2016: First global digital elevation model of Mercury. Lunar Planet. Sci. Conf. 47, \#2959.

Besserer, J., Nimmo, F., Roberts, J. H., Pappalardo, R. T., 2013: Convection-driven compaction as possible origin of Enceladus's long wavelength topography. J. Geophys. Res. 118, doi.org/10.1002/jgre.20079.

Beyer, R. A., Alexandrov, O, McMichael, S., 2018: The Ames Stereo Pipeline: NASA's open source software for deriving and processing terrain data. Earth Space Sci., 5. doi.org/10.1029/2018EA000409.

Bland, M. T., Singer, K. N., McKinnon, W. B., Schenk, P. M., 2012: Enceladus' extreme heat flux as revealed by its relaxed craters. Geophys. Res. Lett. 39, L17204, doi.org/10.1029/2012GL052736.

Bland, M. T., Becker, T. L., Edmundson K. L., Roatsch, Th., Archinal, B., Patterson, G. W., Collins, G. C., Schenk, P. M., Pappalardo, R. T., Cook, D. A., 2018: A new Enceladus global control network, image mosaic, and updated pointing kernels from Cassini's 13-year mission. Earth Space Sci., 5. doi.org/10.1029/2018EA000399.

Brown, D. C., 1958: A solution to the general problem of multiple station analytical stereotriangulation. RCA Data Reduction Technical Report, 43.

Cignoni, P., Callieri, M., Corsini, M., Dellepiane, M., Ganovelli, F., Ranzuglia, G., 2008: Meshlab: An open-source mesh processing tool. Sixth Eurographics Italian Chapter Conference, $129-136$. 
Collins, G. C., Goodman, J. C., 2007: Enceladus' south polar sea. Icarus 189, 72-82, doi.org/10.1016/j.icarus.2007.01.010.

Crow-Willard, E. N., Pappalardo, R. T., 2015: Structural mapping of Enceladus and implication for formation of tectonized regions. J. Geophys. Res. 120, doi.org/10.1002/2015JE004818.

Edmundson, K. L., Cook, D. A., Thomas, O. H., Archinal, B. A., Kirk, R. A., 2012: Jigsaw: The ISIS3 bundle adjustment for extraterrestrial photogrammetry. ISPRS Annals, I-4, 203-208. doi.org/10.5194/isprsannals-I-4-203-2012.

Garcia, P. A., Keszthelyi, L., Becker, T. B., Becker, K., Sides, S., 2015: The present and future of pattern matching in the Integrated Software for Imagers and Spectrometers (ISIS). Lunar Planet. Sci. Conf. 42, \#2782.

Hansen, C. J., Esposito, L., Stewart, A. I. F., Colwell, J., Hendrix, A., Pryor, W., Shemansky, D., West, R., 2006: Enceladus' water vapor plume. Science, 311, 5766, 1422-1425, doi.org/10.1126/science.1121254.

Hemingway, D. J., Mittal, T., 2019: Enceladus's ice shell structure as a window on internal heat production. Icarus 332, 111-131, doi.org/10.1016/j.icarus.2019.03.011.

Kirk, R. L., Howington-Kraus, E., Redding, B., Galuska, D., Hare, T., Archinal, B. A., Soderblom, L. A., Barrett, J. M., 2003: High-resolution topomapping of candidate MER landing sites with Mars Orbiter Camera narrow-angle images. J. Geophys. Res. 108, E12, 8088, doi.org/10.1029/2003JE002131.

Laura, J. R., Hare, T. M., Gaddis, L. R., Fergason, R. L., Skinner, J. A., Hagerty, J. J., Archinal, B. A., 2017: Toward a planetary spatial data infrastructure. ISPRS Int. J. Geo-info., 6, 181, doi.org/10.3390/ijgi6060181.

Laura, J. R., Bland, M. T., Fergason, R. L., Hare, T. M., Archinal, B. A., 2018: Framework for the development of planetary spatial data infrastructures: A Europa case study. Earth Space Sci., 5. doi.org/10.1029/2018EA000411.

McKinnon, W. B., 2015: Effects of Enceladus' rapid synchronous spin on interpretation of Cassini gravity. Geophys. Res. Lett. 42, doi.org/10.1002/2015GL063384.

Meyer, J., Wisdom, J., 2007: Tidal heating in Enceladus. Icarus 188, 535-539, doi.org/10.1016/j.icarus.2007.03.001.

Nimmo, F., Bills, B. G., Thomas, P. C., 2011: Geophysical implications of the long-wavelength topography of the Saturnian satellites. J. Geophys. Res. 106, E11001, doi.org/10.1029/2011JE003835.

Poroc, C. C., West, R. A., Squyres, S., McEwen, A., Thomas, P., Murray, C. D., Delgenio, A., Ingersoll, A. P., Johnson, T., Neukum, G., Veverka, J., Dones, L., Brahic, A., Burns, J. A., Haemmerle, V., Knowles, B., Dawson, D., Roatsch, T., Beurle, K., Owen, W., 2004: Cassini Imaging Science: Instrument characteristics and anticipated scientific investigations at Saturn. Space Sci. Rev., 115, 363-497, doi.org/10.1007/s11214-0041456-7.

Porco, C. C., Helfenstein, P., Thomas, P. C., Ingersoll, A. P., Wisdom, J., West, R., Neukum, G., Denk, T., Wagner, R., Roatsch, T., Kieffer, S., Turtle, E., McEwen, A., Johnson, T.,
Rathbun, J., Veverka, J., Wilson, D., Perry, J., Spitale, J., Brahic, A., Burns, J. A., DelGenio, A. D., Dones, L., Murray, C. D., Squyres, S., 2006: Cassini observes the active south pole of Enceladus. Science, 311, 5766, 1393-1401, doi.org/10.1126/science.1123013.

Postberg, F., Kempf, S., Schmidt, J., Brilliantov, N., Beinsen, A., Abel, B., Buck, W., Srama, R., 2009: Sodium salts in E-ring ice grains from an ocean below the surface of Enceladus. Nature 459, 1098-1101, doi.org/10.1039/nature08046.

Roberts, J. H., Nimmo, F., 2008: Tidal heating and the long-term stability of a subsurface ocean on Enceladus. Icarus 194, 675689, doi.org/10.1016/j.icarus.2007.11.010.

Roberts, J. H., 2015: The fluffy core of Enceladus. Icarus 258, 54-66, doi.org/10.1016/j.icarus.2015.05.033.

Schenk, P. M., McKinnon, W. B., 2009: One-hundred-km-scale basins on Enceladus: Evidence for an active shell. Geophys. Res. Lett., 36, L16202., doi.org/10.1029/2009GL039916.

Souček, O., Běhounková, M., Čedek, O., Hron, J., Tobie, G., Choblët, G., 2019: Tidal dissipation in Enceladus' uneven, fractured ice shell. Icarus 328, 218-231, doi.org/10.1016/j.icarus.2019.02.012.

Spencer, J. R., Pearl, J. C., Segura, M., Flaser, F. M., Mamoutkine, A., Romani, P., Buratti, B. J., Hendrix, A. R., Spilker, L. J., Lopes, R. M. C., 2006: Cassini encounters Enceladus: Background and the discovery of a south polar hot spot. Science, 311, 5766, 1401-1405, doi.org/10.1126/science.1121661.

Tajeddine, R., Soderlund, K. M., Thomas, P. C., Helfenstein, P., Hedman, M. M., Burns, J. A., Schenk, P. M., 2017: True polar wander of Enceladus from topographic data. Icarus 295, 46-60, doi.org/10.1016/j.icarus.2017.04.019.

Thomas, P. C., 2010: Sizes, shapes, and derived properties of the Saturnian satellites after the Cassini nominal mission. Icarus, 208, 395-401, doi.org/10.1016/j.icarus.2010.01.025.

Thomas, P. C., Burns, J. A., Helfenstein, P., Squyres, S., Veverka, J., Porco, C., Turtle, E. P., McEwen, A., Denk, T., Giese, B., Roatsch, T., Johnson, T. V., Jacobson, R. A., 2007: Shapes of the saturnian satellites and their significance. Icarus 190, 573-584, doi.org/10.1016/j.icarus.2007.03.012.

Thomas, P. C., Tajeddine, R., Tiscareno, M. S., Burns, J. A., Joseph, J., Loredo, T. J., Helfenstein, P., Porco, C. C., 2016: Enceladus's measured physical libration requires a global subsurface ocean. Icarus 264, 37-47, doi.org/1016/j.icarus.2015.08.037. 